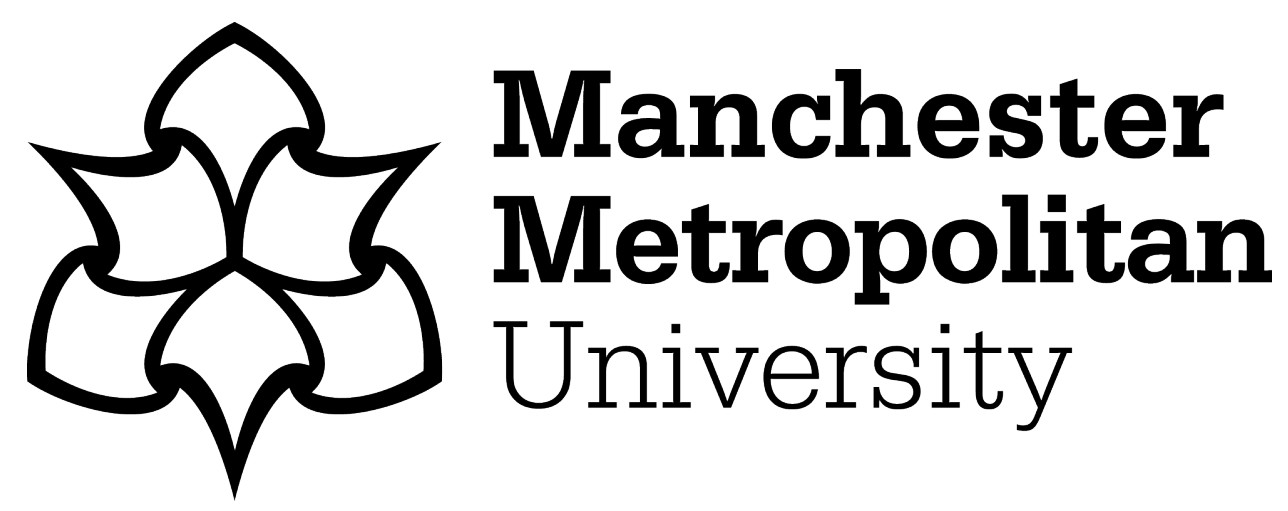

Kislov, Roman ORCID logoORCID: https://orcid.org/0000-0003-2525-7673, Harvey, Gill and Jones, Lorelei (2021) Boundary organising in healthcare: theoretical perspectives, empirical insights and future prospects. Journal of Health Organization and Management, 35 (2). pp. 133-140. ISSN 14777266

Downloaded from: https://e-space.mmu.ac.uk/627644/

Version: Accepted Version

Publisher: Emerald

DOI: https://doi.org/10.1108/jhom-04-2021-475

Please cite the published version 


\title{
Boundary organising in healthcare: Theoretical perspectives, empirical insights and future prospects
}

Roman Kislov, Manchester Metropolitan University and The University of Manchester, UK

Gill Harvey, Flinders University and Queensland University of Technology, Australia

Lorelei Jones, Bangor University, UK

\begin{abstract}
Purpose - To introduce a special issue on boundary organising in healthcare bringing together a selection of six leading papers accepted for presentation at the $12^{\text {th }}$ Organisational Behaviour in Health Care (OBHC 2020) Conference.
\end{abstract}

Design/methodology/approach - In this introductory paper, the guest editors position the special issue papers in relation to the theoretical literature on boundaries and boundary organising and highlight how these contributions advance our understanding of boundary phenomena in healthcare.

Findings - Three strands of thinking - practice-based, systems theory and place-based approaches are briefly described, followed by an analytical summary of the six papers included in the special issue. The papers illustrate how the dynamic processes of boundary organising, stemming from the dual nature of boundaries and boundary objects, can be constrained and enabled by the complexity of broader multi-layered boundary landscapes, in which local clinical and managerial practices are embedded.

Originality/value - We set the scene for the papers included in the special issue, summarise their contributions and implications, and suggest directions for future research.

Research implications/limitations - We call for interdisciplinary and multi-theoretical investigations of boundary phenomena in health organisation and management, with a particular attention to (1) the interplay between multiple types of boundaries, actors and objects operating in complex multilayered boundary systems; (2) diversity of the backgrounds, experiences and preferences of patients and services users; and (3) the role of artificial intelligence and other non-human actors in boundary organising.

Practical implications - Developing strategies of reflection, mitigation, justification and relational work is crucial for the success of boundary organising initiatives.

Keywords: boundaries; boundary organising; boundary management; boundary reconfiguration; boundary objects; boundary spanning; healthcare; practice-based theorising; systems theory; placebased approaches

\section{Introduction}

Contemporary healthcare is characterised by complex structural and functional arrangements, representing a wide range of interrelated professions, organisations and groups operating in diverse geographical and sociocultural contexts. It features numerous boundaries - frontiers, demarcations and distinctions reflecting differences between different domains (Lamont and Molnár, 2002) and 
potentially leading to discontinuities in action or interaction (Akkerman and Bakker, 2011). Boundary organising (Mørk et al., 2012) - identifying and navigating multiple boundaries in this multi-layered landscape - constitutes an important aspect of healthcare management practice. Getting it right is essential for the success of change initiatives at different levels as well as for preventing unwarranted variation in healthcare outcomes across different organisational and regional contexts.

The papers in this special issue, drawing on a diverse range of theoretical perspectives, are united by their interest in an empirical exploration of different aspects of boundary organising. All of them were accepted for presentation at the $12^{\text {th }}$ Organisational Behaviour in Health Care (OBHC 2020) Conference which was scheduled to take place in Manchester, UK, in April 2020 but had to be cancelled due to the Covid-19 pandemic. Bringing these papers together can be seen as a collective act of resistance on the part of editors and contributors, aiming to maintain the continuity of tradition and enable circulation of knowledge despite the reinforced interpersonal, regional and international boundaries imposed by the global health crisis.

We start by briefly describing our overarching approach to conceptualising boundaries and boundary organising. This is followed by outlining three interrelated strands of thinking - practice-based, systems theory and place-based approaches - which are important for positioning the six papers included in the special issue. After that, we describe the contribution each of the papers makes to our understanding of boundary-related phenomena. We conclude by describing the implications of these papers for healthcare management research and practice.

\section{Theoretical perspectives on boundaries and boundary organising}

Prior to describing the intellectual traditions that inspired the papers in this special issue, it is worth highlighting some of the key characteristics of boundaries which are now broadly accepted across the social sciences. First, boundaries are intersubjective, negotiated, and set through the decisions and actions of social actors (Heracleous, 2004), making them inherently arbitrary, open to contestation and intertwined with local and societal power arrangements. Second, boundaries are inherently dual (Akkerman and Bakker, 2011). On the one hand, they often create barriers to the flows of information and other resources, leading to discontinuity and mandating the introduction of boundary crossing interventions. On the other, they suggest potential for continuity by showing the mutual relevance of the interrelated domains they separate and creating conditions for innovative cross-fertilisation between different perspectives. Finally, this socially constructed and dualistic nature of boundaries renders them fluid and dynamic, with their configurations and permeability prone to change over time. The practices of boundary organising therefore involve a fine balance between destabilising (also referred to as spanning, bridging and crossing) and (re)stabilising (buffering or reinforcing) multiple boundaries (Kislov, 2018; Mørk et al., 2012).

A family of practice-based approaches, including but not limited to Bourdieu's theory of practice and Wenger's theory of communities of practice, has been particularly influential in developing our understanding of boundary phenomena in healthcare. Carlile's (2004) widely used framework provides a categorisation of boundaries into syntactic (differences in language), semantic (differences in meaning) and pragmatic (differences in practice) and suggests how these could be dealt with in the process of cross-disciplinary collaboration. A lot of attention has also been paid to the mechanisms of boundary crossing (Akkerman and Bakker, 2011) and the role played by such 'boundary bridges' (Wenger, 1998) as boundary objects (Nicolini et al., 2012) and boundary spanners (also known as knowledge brokers) (Kislov et al., 2017). Due to their focus on boundaries between practices, practice-based approaches are particularly well suited for analysing interactions between different professional or occupational groups co-located in a team and/or organisation, with interorganisational boundaries usually receiving less attention.

The concept of organisational boundaries features more prominently in systems theory which views organisations as clearly demarcated entities interacting with an 'external' environment (Parsons, 
2013 [1951]). An external boundary serves as an important distinction between the system and the environment, making the system unique and giving it definition. The boundary is therefore constitutive of the system, enabling the latter to exist (Cilliers, 2010; Friedman and Allen, 2011; Luhmann, 2006). Boundaries are permeable, enabling the system to secure the necessary resources, selectively respond to environmental perturbations and develop interdependencies with the environment, but their permeability varies (Lai and Lin, 2017). Systems are also internally differentiated, with internal boundaries and diversity constituting the richness of the system and seen as valuable resources reflecting environmental complexity and enabling adaptation and growth in a challenging and changing environment (Cilliers, 2010). Overall, system theory approaches privilege the maintenance of the system and its boundaries (Heracleous, 2004), with less focus on the agency of organisational members and the processes of boundary crossing.

Finally, there is a growing interest in place-based approaches to health policy and delivery, which focus on spatial boundaries, such as neighbourhood or regional borders, and geographical barriers, such as the distance between a consumer and provider of health services (Bissonnette et al., 2012; Wang and Luo, 2005). As the notion of place is reified in maps and policy documents, it is tempting to treat 'place' as something bounded, static and coherent (Hammond et al., 2017). Recent work, however, emphasises the emergent, relational and fluid nature of spatial boundaries, which are produced by a complex web of social relations, interactions and practices and can themselves have social effects (Massey, 2005). From this perspective, boundary organising can be viewed through the notion of assemblage, reflecting ongoing practices of assembling, disassembling and reassembling a range of heterogeneous actors, resources or policies to create new 'carescapes' (Ivanova et al., 2016; Lorne et al., 2019). By juxtaposing spatial, social and other types of boundaries, this line of enquiry can be fruitful for exploring complex, multi-layered boundary systems.

In different ways, each of the papers included in this special issue engages with one or more of the approaches described above, combining theoretical grounding with empirical focus. In their study informed by practice-based theorising, Sharp and colleagues (2021) deploy the concept of boundary object to explore development of a smartphone app. Allen (2021) applies Translational Mobilisation Theory (Allen, 2018), also grounded in practice-based approaches, to theorise how an organisational understanding of patients is accomplished in an emergency services control room. Rowland and colleagues (2021) examine patient engagement programmes, focusing on metaphors of organisations as boundary bridges and contributing insights about perceptions of organisations as bounded systems. Luhmann's $(2006,2018)$ systems theoretical approach is used by Jansson and colleagues (2021) to reveal strategies of justification involved in making and legitimating decisions about a hybrid hospital unit. The last two papers engage with place-based approaches, with Carlsson and Pijpers (2021) developing a relational perspective on neighbourhood governance and Schuurmans et al. (2021) exploring the formation and composition of 'regions' as places of care. Detailed summaries of these contributions are presented in the following section.

\section{Papers included in the special issue and their empirical insights}

We open the special issue with a paper deploying the concept of boundary objects to examine the interactional and contextual factors that shape the development of a technological innovation in healthcare and the implications for adoption and change (Sharp et al., 2021). Using a longitudinal case study approach, Sharp and colleagues follow the co-development, adoption and implementation of a smartphone app to facilitate real-time monitoring of people with rheumatoid arthritis in the English health care system. The analysis illustrates the operation of the app as a boundary object and shows how the outputs from the app create so-called secondary boundary objects, an example being the detailed graphical accounts produced by the app to map disease activity between clinical consultations. In this way, a system of inter-connected boundary objects brings together patients, professionals and managerial groups involved in the process of developing and applying the app. However, this network of boundary objects can simultaneously enable and 
inhibit collaboration between the different actors, illustrating the dual nature of boundaries. On the one hand, the app enabled integration through enhancing two-way knowledge flow between the patient and the clinician, as well as changing the nature of the consultation through opening up broader areas for discussion. On the flip side, this was where the app also demonstrated its disruptive potential because it changed established power relations and dynamics between patients and clinicians, which could pose a barrier to wider diffusion. From a boundary organising perspective, this serves to illustrate how the development of a boundary object is embedded within and shaped by what the authors describe as a 'constellation of social interests, relations and perspectives brought by each community of practice to the project'. It also demonstrates the importance of agency, with a need for human interaction to support the functioning of a boundary object in practice.

One way that patients cross the threshold of healthcare systems is as an emergency, following an injury or the onset or deterioration of illness requiring urgent medical treatment. When a member of the public calls an ambulance their problem must be translated into a response category; 'red' (immediately life threatening), 'amber' (serious but not immediately life threatening, but urgent and may need care at the scene) or 'green' (not serious or life threatening and may be managed by other health services). Allen's (2021) ethnographic study, based on time spent shadowing call operators in an ambulance control centre in Wales, explores this process of 'patient making' at the boundary of the healthcare system. Allen applies Translational Mobilisation Theory, a practice theory that explains the coordination of collective action focusing on the interactions of people, materials and technology (Allen, 2018). In the ambulance call centre a key actor was the computerised Medical Priority Dispatch System. An example of a decision support tool built on abstract universalised rules and algorithms, it was designed to enable non-clinical staff to apply the response categories in a standardised way. Allen shows what happens when the rational, individualist assumptions of the medical priority dispatch system come face to face with the emotional and social narratives of callers. The scripted questions and fixed ordering constrain interaction so that the call handler must discipline the caller to accomplish the work of making and moving patients. This illustrates how organisational understandings of patients and their individual troubles are transformed into 'objects of practice' at service interfaces.

By contrast, Rowland and colleagues (2021) examine how the notion of 'organisation' can be coconstructed by patients and organisational members taking part in patient engagement activities. The novelty of their study for analysing cross-boundary phenomena in healthcare lies in highlighting the possibility of different sense-making frames serving as semantic boundaries that need to be navigated throughout the engagement work. Based on interviews with the participants of patient engagement programmes in two Canadian healthcare providers, the authors present a range of metaphors of the organisation, including organisations as (1) power and politics, (2) bounded systems, and (3) narratives. These metaphors highlight that patient engagement programmes can be viewed as contested liminal spaces open to a variety of interpretations, with lines of contestation not necessarily reflecting the divide between professionals and the public. Of particular interest to the topic of this special issue is the ambiguous liminal position of patient representatives who, especially in the case of successful patient engagement, can be seen as 'too internal' to the organisation, possibly to the detriment of 'outward' engagement activities or democratic representation values. Other important questions arising from this paper are about the extent to which the liminal space of patient engagement allows co-construction of shared cross-boundary knowledge and the degree to which the dissenting patient voices may be at risk of being silenced by recourse to dominant organisational narratives and values. The authors conclude by questioning the existence of 'a single, unified patient voice positioned outside of the organisation' and suggest that 'metaphors-in-use' could be used to explore how the multiplicity of patient voices is assembled and re-assembled as part of ongoing boundary work characterising patient engagement programmes. 
The paper by Jansson and colleagues (2021) takes us further on a journey of exploring boundary processes at the organisational level. It offers an analysis of hybridisation taking place between different social domains, such as healthcare, science, education and law, in a newly established organisational unit within a Finnish hospital. The organisational focus of this qualitative study is well served by adopting a systems theoretical perspective, namely Luhmann's $(2006,2018)$ theory of organisations as self-reproducing systems of communications. This approach views organisations as demarcating themselves from the environment through their 'decision communications'. The latter are inherently paradoxical and self-contradictory as they simultaneously (but often implicitly) contain information about a particular decision; alternatives to that decision; and the reasons, or justifications, behind adopting the actual decision and discarding its alternatives (Cooren and Seidl, 2020). The authors describe three novel justification strategies (justification by problems, justification by examples, and justification by obligations) which can be deployed in hybrid organisational contexts that lack, due to their emerging nature, previously agreed 'decision premises', such as codified strategies or formal rules. By concealing the paradoxical nature of decision-making and enhancing acceptability and legitimacy of decisions, these justification strategies internalise environmental complexity, provide points of reference for further decisionmaking and, ultimately, contribute to institutionalisation of novel hybrid arrangements. In addition, these strategies involve making references to broader societal domains, which reinforces structural coupling between the hybrid organisation and its environment and, due to the multiplicity of said domains, imparts certain flexibility to the process of justification.

Carlsson and Pipers (2021) adopt a place-based approach to studying boundaries, with a specific focus on the neighbourhood governance of social care and how this affects the ability of front-line workers to address health inequities faced by older ethnic minority groups. The authors present findings from a two-year multiple case study undertaken in two different cities in the Netherlands, where they examined policy and practice relevant to older ethnic minority populations. Through a series of interviews with key stakeholders and extensive participant observation, the study explores issues relating to the social and cultural distance to social care and efforts to bridge this distance through relational work. As such, the emphasis is on the socio-relational barriers to care, as opposed to the physical distance between people and services. Whilst neighbourhood governance concerned with collective decision-making and public service delivery at the sub-local level - has become an increasingly popular policy instrument designed to improve access to, and the delivery of, public services, the authors argue that it is not the spatial proximity per se that generates change. Rather, spatial proximity enables front-line workers to engage in relational work, which at times may involve spanning neighbourhood boundaries to connect with key individuals and groups outside the immediate neighbourhood. From a boundary perspective, such relational work serves as a bridging function that has the potential to achieve equity. The authors conclude that neighbourhood governance can only improve health equity in so far as it allows front-line workers to mobilise local knowledge and multi-professional networks to tailor services to disadvantaged populations in the neighbourhood. This further highlights the fluidity of boundaries and the importance of relational agency to understand, interpret and bridge dynamic boundaries.

The final paper by Schuurmans et al. (2021) takes us from neighbourhoods to regions. Its starting point is that healthcare domains can have multiple boundaries - administrative, geographic, and social - that coexist, overlap and at times conflict. Responding to the renewed policy interest in regions as places of care, the authors ask 'what is a region?', comparing formal and informal networks in an empirical study of care for older people in the Netherlands. While policy conceives regions as a clearly bounded geographic space, Schuurmans and colleagues combine insights from social geography and valuation studies to offer an alternative understanding of regions as fragile and dynamic assemblages of heterogenous institutional actors (e.g. public and private organisations), resources and policies. To understand how these socio-spatial formations are assembled and disassembled, the authors deploy the concept of valuation regimes, i.e. relatively durable discourses, 
practices and infrastructures which institutional actors draw upon to decide how worth is ascribed and argued for in a concrete evaluation (Fochler et al., 2016). They show that healthcare governance in the Netherlands is shaped by the co-existence of multiple conflicting regimes of valuation which may have opposing effects on the formation and composition of regional networks. The authors conclude that calls for closer regional collaboration are not necessarily effective in contexts characterised by the predominance of market evaluation principles which favour competition over collaboration.

\section{Concluding remarks: implications for research and practice}

The papers presented in this special issue further develop our understanding of boundary organising in healthcare. In many ways, malleability of boundaries to repeated cycles of reconfiguration is a direct consequence of their dual, selectively permeable nature: boundaries and boundary bridges can serve, often at the same time, both as enablers of and barriers to knowledge sharing, collaborative working and diffusion of innovation. In addition, processes unfolding at local boundaries of practice are shaped by the features of technology increasingly deployed in contemporary healthcare (Allen, 2021; Sharp et al., 2021) as well as by existing governance and accountability arrangements (Carlsson and Pijpers, 2021; Schuurmans et al., 2021). Due to their highly reified nature, these factors may significantly constrain the agency of actors involved in boundary management. On the other hand, it may be useful to consider local boundary phenomena as part of complex, multi-layered boundary landscapes in which different types of boundaries, such as spatial, administrative, professional, organisational and cultural, not only co-exist, but overlap and conflict. The emergent, pluralistic, and inconsistent nature of these complex boundary systems can be creatively exploited by local actors, who can choose from a range of boundary objects (Sharp et al., 2021), sense-making devices (Rowland et al., 2021), justification strategies (Jansson et al., 2021) and evaluative principles (Schuurmans et al., 2021) to realise their aspirations.

Future research could explore how the interplay between multiple types of boundaries, actors and objects operating in these complex boundary systems affect the organisation and management of healthcare, potentially drawing on the existing socio-material and socio-spatial lenses as well as developing new multi-theoretical and interdisciplinary approaches. As shown by Allen's (2021) case study, a computerised decision-making system can be a central actor at a boundary crossing, and rapid technological advances necessitate further research into the role of non-human actors, such as artificial intelligence, in boundary organising. Another avenue for investigation concerns multiple roles played by the patients and service users. While these groups are at risk of being dehumanised and reduced to boundary objects (Allen, 2021), they can also play a more active role in boundary interactions with health professions and organisations (Sharp et al., 2021). As highlighted by Rowland et al. (2021) and Carlsson and Pijpers (2021), patients and service users should not be treated as a homogeneous group having a unified 'voice', and future research should explore the impact their diverse backgrounds, experiences and preferences may have on boundary organising.

In terms of practical implications for healthcare managers involved in boundary reconfigurations, this special issue highlights the importance of ongoing reflection (Allen, 2021; Rowland et al., 2021), mitigation (Allen, 2021; Sharp et al., 2021), justification (Jansson et al., 2021; Schuurmans et al., 2021) and relational work (Carlsson and Pijpers, 2021; Schuurmans et al., 2021). Reflection should aim at 'diagnosing' the boundary landscape, paying attention to discontinuities caused by the operation of particular boundaries and boundary objects as well as to misalignments between policies, governance structures and realities of work practice on the ground. This should ideally lead to the development of mitigation strategies aiming to address, even if not fully resolve, the most pertinent discontinuities and misalignments. These may involve tapping into pre-existing social networks, securing support of leaders keen to play a boundary spanning role, and building on shared understandings of identity, place and what is important. To legitimise these strategies and reduce potential resistance, it is vital to ensure that all decisions involved in boundary organising 
interventions are explicitly justified, exploring multiple opportunities offered by complex boundary systems. Finally, as boundary organising is an inherently collective phenomenon (Kislov et al., 2017), its success is impossible without relational work, involving a concerted effort to build relationships and alliances with a wide range of stakeholders across boundaries and borders.

\section{Acknowledgements}

RK is part-funded by the National Institute for Health Research Applied Research Collaboration (NIHR ARC) Greater Manchester. The views expressed in this publication are those of the authors and not necessarily those of the National Institute for Health Research or the Department of Health and Social Care.

\section{References}

Akkerman, S. F., \& Bakker, A. (2011). Boundary crossing and boundary objects. Review of Educational Research, 81(2), 132-169.

Allen, D. (2018). Translational mobilisation theory: a new paradigm for understanding the organisational elements of nursing work. International Journal of Nursing Studies, 79, 36-42.

Allen, D. (2021). Prioritising the mobilisation of emergency medical services: patient making at the healthcare gateway. Journal of Health Organization and Management, published online before print, 10.1108/jhom-07-2020-0305, this issue.

Bissonnette, L., Wilson, K., Bell, S., \& Shah, T. I. (2012). Neighbourhoods and potential access to health care: The role of spatial and aspatial factors. Health \& Place, 18(4), 841-853.

Carlile, P. R. (2004). Transferring, translating, and transforming: An integrative framework for managing knowledge across boundaries. Organization Science, 15(5), 555-568.

Carlsson, H., \& Pijpers, R. (2021). Working towards health equity for ethnic minority elders: spanning the boundaries of neighbourhood governance. Journal of Health Organisation and Management, published online before print, 10.1108/jhom-08-2020-0325, this issue.

Cilliers, P. (2010). Difference, Identity and Complexity. In P. Cilliers \& R. Preiser (Eds.), Complexity, Difference and Identity: An Ethical Perspective (pp. 3-18). Dordrecht: Springer Netherlands.

Cooren, F., \& Seidl, D. (2020). Niklas Luhmann's radical communication approach and its implications for research on organizational communication. Academy of Management Review, 45(2), 479-497.

Fochler, M., Felt, U., \& Müller, R. (2016). Unsustainable Growth, Hyper-Competition, and Worth in Life Science Research: Narrowing Evaluative Repertoires in Doctoral and Postdoctoral Scientists' Work and Lives. Minerva, 54(2), 175-200.

Friedman, B. D., \& Allen, K. N. (2011). Systems theory. Theory \& Practice in Clinical Social Work, 2(3), 3-20.

Hammond, J., Lorne, C., Coleman, A., Allen, P., Mays, N., Dam, R., . . Checkland, K. (2017). The spatial politics of place and health policy: Exploring Sustainability and Transformation Plans in the English NHS. Social Science and Medicine, 190, 217-226.

Heracleous, L. (2004). Boundaries in the study of organization. Human Relations, 57(1), 95-103.

Ivanova, D., Wallenburg, I., \& Bal, R. (2016). Care in place: A case study of assembling a carescape. Sociology of Health and IIIness, 38(8), 1336-1349.

Jansson, K., Tuunainen, J., \& Mainela, T. (2021). Concealing paradoxes in decision-making during hospital hybridization - A systems theoretical analysis. Journal of Health Organization and Management, published online before print, 10.1108/JHOM-08-2020-0334, this issue.

Kislov, R. (2018). Selective permeability of boundaries in a knowledge brokering team. Public Administration, 96(4), 817-836.

Kislov, R., Hyde, P., \& McDonald, R. (2017). New game, old rules? Mechanisms and consequences of legitimation in boundary spanning activities. Organization Studies, 38(10), 1421-1444. 
Lai, C.-H., \& Lin, S. H. (2017). Systems Theory. In C. R. Scott, J. R. Barker, T. Kuhn, J. Keyton, P. K. Turner \& L. K. Lewis (Eds.), The International Encyclopedia of Organizational Communication. Oxford: Wiley.

Lamont, M., \& Molnár, V. (2002). The study of boundaries in the social sciences. Annual Review of Sociology, 28, 167-195.

Lorne, C., McDonald, R., Walshe, K., \& Coleman, A. (2019). Regional assemblage and the spatial reorganisation of health and care: the case of devolution in Greater Manchester, England. Sociology of Health and IIIness, 41(7), 1236-1250.

Luhmann, N. (2006). System as difference. Organization, 13(1), 37-57.

Luhmann, N. (2018). Organization and Decision. Cambridge: Cambridge University Press.

Massey, D. B. (2005). For space. London: Sage Publications.

Mørk, B. E., Hoholm, T., Maaninen-Olsson, E., \& Aanestad, M. (2012). Changing practice through boundary organizing: A case from medical R\&D. Human Relations, 65(2), 263-288.

Nicolini, D., Mengis, J., \& Swan, J. (2012). Understanding the role of objects in cross-disciplinary collaboration. Organization Science, 23(3), 612-629.

Parsons, T. (2013 [1951]). The Social System. London: Routledge.

Rowland, P., Fancott, C., \& Abelson, J. (2021). Metaphors of organisations in patient involvement programs: Connections and Contradictions. Journal of Health Organization and Management, published online before print, this issue.

Schuurmans, J. J., van Pijkeren, N., Bal, R., \& Wallenburg, I. (2021). Regionalization in elderly care: what makes up a healthcare region? Journal of Health Organization and Management, published online before print, 10.1108/jhom-08-2020-0333, this issue.

Sharp, C. A., Bresnen, M., Austin, L., McCarthy, J., Dixon William, G., \& Sanders, C. (2021). Implementing disruptive technological change in UK healthcare: exploring development of a smart phone app for remote patient monitoring as a boundary object using qualitative methods. Journal of Health Organisation and Management, published online before print, 10.1108/jhom-07-2020-0295, this issue.

Wang, F., \& Luo, W. (2005). Assessing spatial and nonspatial factors for healthcare access: towards an integrated approach to defining health professional shortage areas. Health \& Place, 11(2), 131-146.

Wenger, E. (1998). Communities of Practice: Learning, Meaning and Identity. Cambridge: Cambridge University Press. 